\title{
On the Zeros of Euler Product Dirichlet Functions
}

\author{
Dorin Ghisa \\ York University, Toronto, Canada \\ Email:dghisa@yorku.ca
}

How to cite this paper: Ghisa, D. (2019) On the Zeros of Euler Product Dirichlet Functions. Advances in Pure Mathematics, 9, 959-966.

https://doi.org/10.4236/apm.2019.912048

Received: October 21, 2019

Accepted: December 7, 2019

Published: December 10, 2019

Copyright (C 2019 by author(s) and Scientific Research Publishing Inc. This work is licensed under the Creative Commons Attribution International License (CC BY 4.0).

http://creativecommons.org/licenses/by/4.0/

\begin{abstract}
We study a class of Dirichlet functions obtained as analytic continuation across the line of convergence of Dirichlet series which can be written as Euler products. This class includes that of Dirichlet L-functions. The problem of the existence of multiple zeros for this last class is outstanding. It is tacitly accepted, yet not proved that the Riemann Zeta function, which belongs to this class, does not possess multiple zeros. In a previous study we provided an example of Dirichlet function having double zeros, but that function is not an Euler product function. In this paper we deal with Euler product functions and by using the geometric properties of the mapping realized by these functions, we tackle the problem of the multiplicity of their zeros.
\end{abstract}

\section{Keywords}

General Dirichlet Series, Euler Products, Fundamental Domains, Analytic Continuation

\section{Introduction}

We are dealing with normalized series of the form:

$$
\zeta_{A, \Lambda}(s)=\sum_{n=1}^{\infty} a_{n} e^{-\lambda_{n} s}=1+a_{2} e^{-\lambda_{2} s}+\cdots
$$

where $A=\left\{a_{1}=1, a_{2}, \cdots\right\}$ is an arbitrary sequence of complex numbers (the coefficients of the series) and $\Lambda=\left\{\lambda_{1}=0, \lambda_{2}, \cdots\right\}$ is a sequence of non decreasing real numbers (the exponents of the series).

When $\lambda_{n}=\ln n$, then the series has the form $\zeta_{A, \Lambda}(s)=\sum_{n=1}^{\infty} \frac{a_{n}}{n^{s}}$ and it is called ordinary Dirichlet series. There is a vast literature on general Dirichlet series, a part of which has been mentioned in [1] [2] where we brought contributions to different aspects of this topic. In this paper we deal only with 
the problem of the multiplicity of zeros of Dirichlet functions and it is sufficient to study those two papers in order to get the necessary information supporting the development below.

We suppose that the function $a(n)=a_{n}$ is totally multiplicative, i.e. $a(m n)=a(m) a(n)$ for every $m, n \in \mathbb{N}$. Then for every prime decomposition $n=n_{1}^{k_{1}} n_{2}^{k_{2}} \cdots n_{j}^{k_{j}}$ we have $a(n)=a\left(n_{1}\right)^{k_{1}} a\left(n_{2}\right)^{k_{2}} \cdots a\left(n_{j}\right)^{k_{j}}$.

If the series is an ordinary one, then

$$
\begin{aligned}
\lambda(n) & =\lambda_{n}=\ln n=k_{1} \ln n_{1}+k_{2} \ln n_{2}+\cdots+k_{j} \ln n_{j} \\
& =k_{1} \lambda\left(n_{1}\right)+k_{2} \lambda\left(n_{2}\right)+\cdots+k_{j} \lambda\left(n_{j}\right) .
\end{aligned}
$$

Assuming that we have also this last property for a general Dirichlet series, then it can be easily shown (see [1], theorem 11) that the series appears also as an Euler product:

$$
\zeta_{A, \Lambda}(s)=\prod_{p \in \mathfrak{\wp}}\left(1-a_{p} e^{-\lambda_{p} s}\right)^{-1}
$$

where $\wp$ is the set of prime numbers. For such a series we have

$$
\frac{\zeta_{A, \Lambda}^{\prime}(s)}{\zeta_{A, \Lambda}(s)}=-\sum_{p \in \mathfrak{A}} \lambda_{p} a_{p} e^{-\lambda_{p} s} /\left(1-a_{p} e^{-\lambda_{p} s}\right)
$$

The series on the left hand side of this equality have the same half plane of convergence which must coincide with that of the right hand side.

We denote by $e^{\Lambda}$ the sequence $\left\{e^{\lambda_{1}}, e^{\lambda_{2}}, \cdots\right\}$.

It is known that (see [1]) if $\zeta_{A, \Lambda}(s)$ has a finite abscissa of convergence, then the abscissa of convergence of $\zeta_{A, e^{\Lambda}}(s)$ is zero.

Moreover, if $\zeta_{A, e^{\wedge}}(s)$ has a discrete set of singular points on the imaginary axis, then $\zeta_{A, \Lambda}(s)$ can be continued as a meromorphic function in the whole complex plane. Dirichlet $L$-series can be continued in this way.

Suppose that the series $\zeta_{A, \Lambda}(s)$ admits such a continuation and satisfies (2). We will call the extended function Euler product Dirichlet function.

Meromorphic continuations are possible also for the series $\zeta_{A, \Lambda}^{\prime}(s)$ and for the right hand side term in (3).

Let us denote by $\varphi(s)$ the meromorphic function obtained on the right hand side in (3) by this continuation.

By the uniqueness theorem of analytic functions, $\varphi(s)$ coincides with $\frac{\zeta_{A, \Lambda}^{\prime}(s)}{\zeta_{A, \Lambda}(s)}$; therefore it can have only simple poles.

\section{The Geometry of the Mappings by General Dirichlet Series}

From (1) it can be easily seen that $\lim _{\sigma \rightarrow+\infty} \zeta_{A, \Lambda}(\sigma+i t)=1$. We have shown in [2] that this happens uniformly with respect to $t$.

This simple fact has important consequences regarding the landscape of the pre-image of the real axis by a Dirichlet function (see Figure 1). A similar landscape is produced by the pre-image of the real axis by the derivative of $\zeta_{A, \Lambda}(s)$ (see Figure 2). 


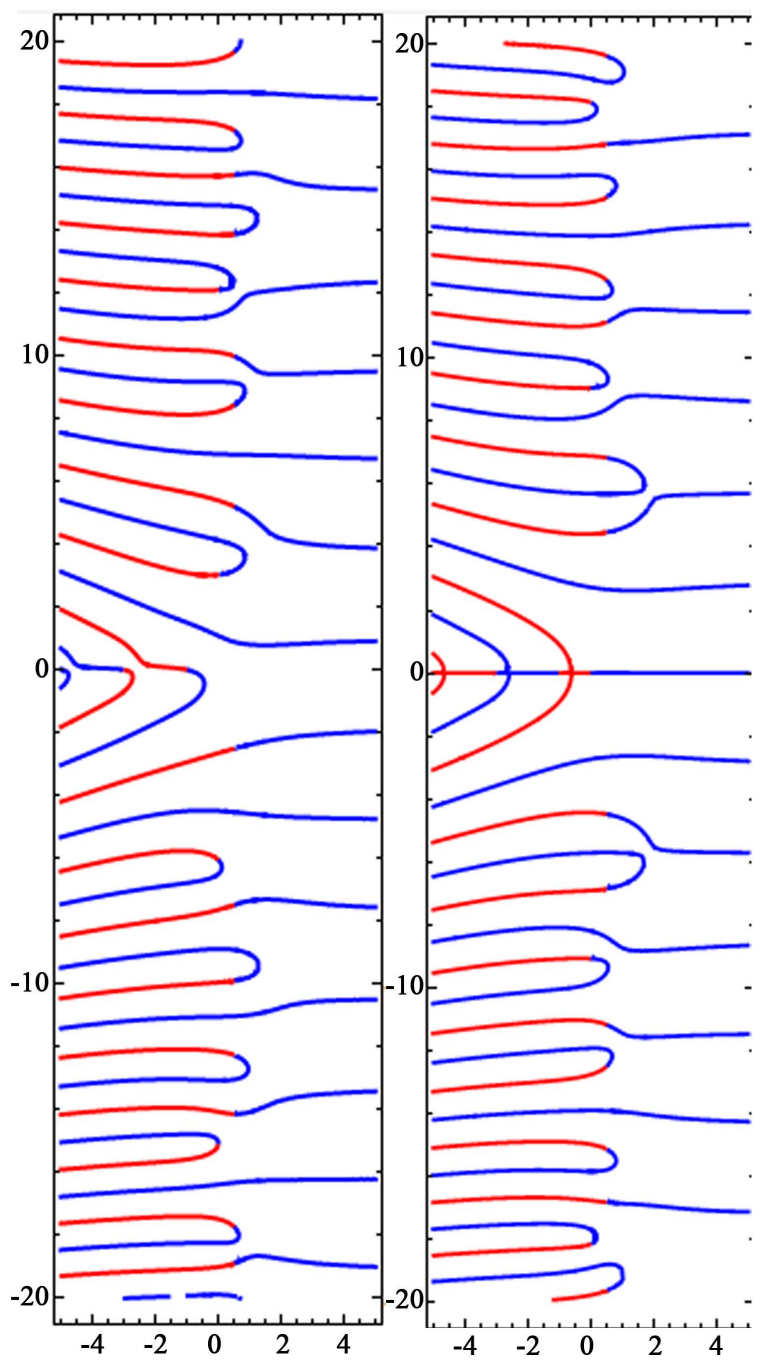

Figure 1. The landscape of the pre-image of the real axis by Dirichlet L-functions defined by a complex and respectively a real Dirichlet character.

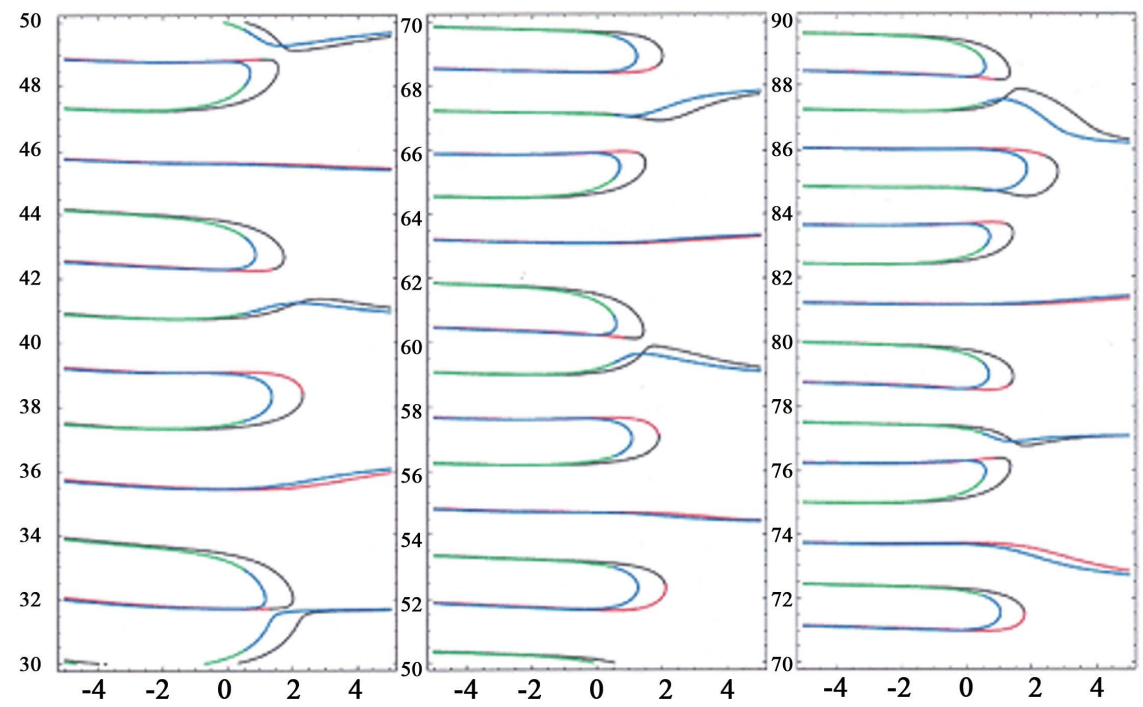

Figure 2. A sample of intertwining curves of the Riemann Zeta function. 
Let us list a few facts valid for any Dirichlet function. The proofs can be found in [1] [2].

Proposition 2.1: The pre-image of the real axis by $\zeta_{A, \Lambda}(s)$ contains infinitely many disjoint curves $\Gamma_{k}^{\prime}$ extending for $\sigma$ from $-\infty$ to $+\infty$ which are mapped each one bijectively by $\zeta_{A, \Lambda}(s)$ onto the interval $(1,+\infty)$ of the real axis. Consecutive curves $\Gamma_{k}^{\prime}$ and $\Gamma_{k+1}^{\prime}$ form infinite strips $S_{k}$, $k \in \mathbb{Z}$, where $S_{0}$ contains the real axis.

Proposition 2.2: Every strip $S_{k}, k \neq 0$ contains a unique component $\Gamma_{k, 0}$ of the pre-image of the real axis which is mapped bijectively by $\zeta_{A, \Lambda}(s)$ onto the interval $(-\infty, 1)$ and a unique unbounded component of the pre-image of the unit circle. It contains also a finite number of curves $\Gamma_{k, j}, j \neq 0$ which are mapped bijectively onto the whole real axis. Every curve $\Gamma_{k, j}$ contains a unique zero of $\zeta_{A, \Lambda}(s)$.

Proposition 2.3: The curves $\Gamma_{k, j}$ are disjoint, except that $\Gamma_{k, 0}$ can meet $\Gamma_{k, 1}$ or $\Gamma_{k,-1}$ into a double zero of $\zeta_{A, \Lambda}(s)$.

Proposition 2.4: If we color, for example, red the pre-image of the negative real half axis and blue the pre-image of the positive real half axis, then the pre-image of any circle centered at the origin will meet alternatively the color red and the color blue. This is the color alternating rule. It is illustrated in Figure 3. The same rule applies also to the pre-image of the real axis by any derivative of $\zeta_{A, \Lambda}(s)$.

Proposition 2.5: When represented in the same plane, the pre-image of the real axis by both $\zeta_{A, \Lambda}(s)$ and $\zeta_{A, \Lambda}^{\prime}(s)$, come in couples of curves $\Gamma_{k}^{\prime}$ and $\Upsilon_{k}^{\prime}$, respectively $\Gamma_{k, j}$ and $\Upsilon_{k, j}$ (see [2], Theorem 4) which intersect two by two (the intertwining curves).

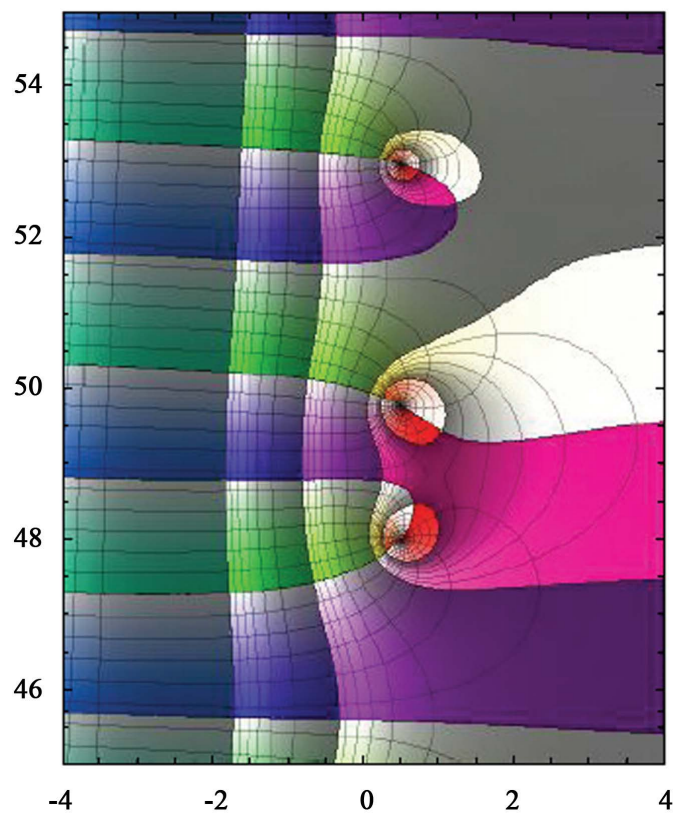

Figure 3. An illustration of the color alternating rule. The vertical lines and the lines around zeros are pre-images of circles centered at the origin. 
Proposition 2.6: The intertwining curves intersect each other in points where the tangent to $\Gamma_{k, j}$, respectively $\Gamma_{k}^{\prime}$ is horizontal, or in multiple zeros, where the tangents do not exist. An illustration of this fact can be seen in Figure 2 above.

Proposition 2.7: If we denote by a the color of the pre-image of the negative real half axis by $\zeta_{A, \Lambda}(s)$ and $\mathbf{b}$ that of the positive real half axis and by $\mathbf{c}$ and $\mathbf{d}$ the colors of the pre-image of the same half axes by $\zeta_{A, \Lambda}^{\prime}(s)$, then color a can meet only color $\mathbf{d}$ and color $\mathbf{b}$ can meet only color $\mathbf{c}$, except for the case of $\Gamma_{k, 0}$ and $\Upsilon_{k, 0}$, where color $\mathbf{d}$ meets both color $\mathbf{a}$ and $\mathbf{b}$. This is the color matching rule.

We notice that there is no exception to this rule when instead of the couple $\zeta_{A, \Lambda}(s)$ and $\zeta_{A, \Lambda}^{\prime}(s)$ we take the couple $\zeta_{A, \Lambda}^{\prime}(s)$ and $\zeta_{A, \Lambda}^{\prime \prime}(s)$ since $\Upsilon_{k, 0}$ does not change color. The same thing can be said for any couple of consecutive derivatives of $\zeta_{A, \Lambda}(s)$.

Proposition 2.8: The color matching rule forbids double zeros at the intersection of $\Gamma_{k, j}$ and $\Upsilon_{k, j}$ when $j \neq 0$.

Proposition 2.9: If $s_{k, j}$ are the zeros of $\zeta_{A, \Lambda}^{\prime}(s)$ in $S_{k}$, then the pre-image of the segment $l_{k, j}$ from $z=1$ to $z=\zeta_{A, \Lambda}\left(s_{k, j}\right)$ and the curves $\Gamma_{k, j}$ and $\Gamma_{k}^{\prime}$ bound fundamental domains which are mapped conformally by $\zeta_{A, \Lambda}(s)$ onto the whole complex plane with slits alongside these segments and the interval $[1,+\infty)$ of the real axis. In a similar way fundamental domains are obtained also for $\zeta_{A, \Lambda}^{\prime}(s)$.

\section{Local Mapping Properties of Analytic Functions}

It is known (see [3], page 133):

Proposition 3.1: If $z_{0}$ is a regular point of the analytic function $f(z)$, then in a neighborhood of $z_{0}$ we have:

$$
f(z)=f\left(z_{0}\right)+\left(z-z_{0}\right)^{n} h(z)
$$

where $n$ is a positive integer and $h(z)$ is analytic at $z_{0}$ and $h\left(z_{0}\right) \neq 0$

Depending on the value of $n$, the local mapping by $f(z)$ at $z_{0}$ has the form seen in Figure 4.

Here the arcs $\gamma: s=s(x)$ are mapped by $f(z)$ onto the interval $(0, r)$, where $r$ is the radius of the image disc, such that $f(s(x))=x$.

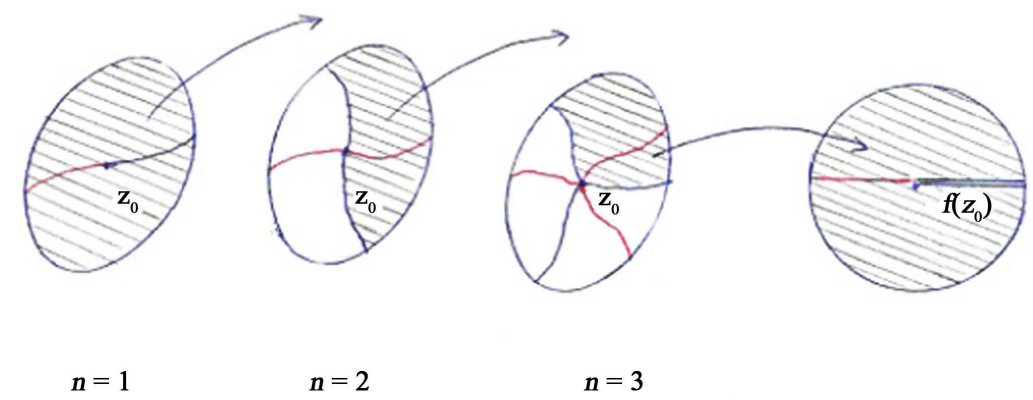

Figure 4. The local mapping by an analytic function at a regular point. 
They are analytic arcs (see [3], page 234) i.e. the derivative $s^{\prime}(x)$ exists on $(0, r)$. Moreover, $\lim _{x \backslash 0} s^{\prime}(x)$ exists. The angles at $s_{0}$ are doubled, tripled, etc. by $f(z)$ according with $n=2, n=3$, etc. and they remain the same when $n=1$.

\section{The Multiplicity of the Zeros of $\zeta_{A, \Lambda}(s)$}

We proved in [2] that linear combinations of linearly independent Dirichlet functions satisfying the same Riemann type of functional equation have double zeros.

If $f_{\tau}(s)=(1-\tau) f_{1}(s)+\tau f_{2}(s), 0 \leq \tau \leq 1$ is such a linear combination, the when $\tau$ varies from 0 to 1 the zeros of $f_{1}(s)$ move continuously to the zeros of $f_{2}(s)$.

When a couple of zeros of $f_{1}(s)$ symmetric with respect to the critical line move to a couple of zeros of $f_{2}(s)$ on the critical line, there must be a value of $\tau$ for which the two zeros coincide, and therefore $f_{\tau}(s)$ has a double zero.

An illustration of such a zero can be seen in Figure 5 .

These linear combinations cannot be Euler product functions. We will deal next with Euler product Dirichlet functions.

Theorem 4.1 Euler product Dirichlet functions do not have any multiple zero.

Proof: Suppose that $\zeta_{A, \Lambda}(s)$ is an Euler product Dirichlet function. Then the formula (3) is valid for $\zeta_{A, \Lambda}(s)$, where the function $\varphi(s)$ is meromorphic in the whole plane and has only simple poles. Indeed, by the section 3 at any point $s_{0}$ the function $\zeta_{A, \Lambda}(s)$ has the form $\zeta_{A, \Lambda}(s)=\left(s-s_{0}\right)^{m} g(s)$, where $m \in \mathbb{Z}$ and $g(s)$ is analytic at $s_{0}$ and $g\left(s_{0}\right) \neq 0$.

When $m=0$, the function $\zeta_{A, \Lambda}(s)$ is analytic at $s_{0}$ and $\zeta_{A, \Lambda}\left(s_{0}\right) \neq 0$, therefore $\varphi(s)$ is analytic at $s_{0}$. When $m \neq 0$ then $\zeta_{A, \Lambda}^{\prime}(s)=m\left(s-s_{0}\right)^{m-1} g(s)+\left(s-s_{0}\right)^{m} g^{\prime}(s)$, hence $\varphi(s)=\frac{m}{s-s_{0}}+\frac{g^{\prime}(s)}{g(s)}$, where $g\left(s_{0}\right) \neq 0$, thus $\varphi(s)$ has a simple pole at $s_{0}$.

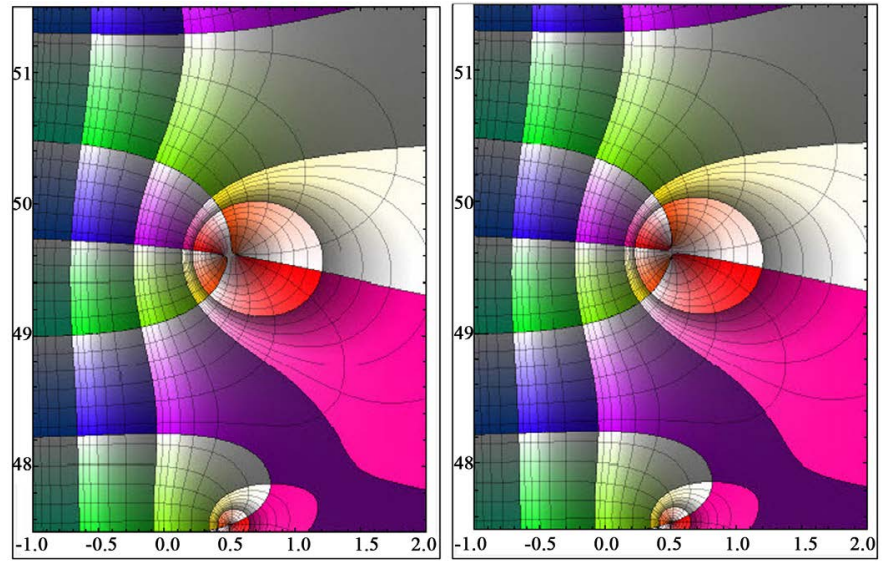

Figure 5. The zeros of $f_{\tau}(s)$ for two close values of $\tau$. They must coincide for an intermediate $\tau$. 
Let us assume that $s_{0}$ is a multiple zero of $\zeta_{A, \Lambda}(s)$, i.e. $\zeta_{A, \Lambda}\left(s_{0}\right)=\zeta_{A, \Lambda}^{\prime}\left(s_{0}\right)=0$. Then, when trying to compute $\varphi\left(s_{0}\right)$ we get an indetermination of the form $0 / 0$. The l'Hospital rule is applicable, and $\varphi\left(s_{0}\right)=\lim _{s \rightarrow s_{0}} \frac{\zeta_{A, \Lambda}^{\prime \prime}(s)}{\zeta_{A, \Lambda}^{\prime}(s)}$. If $\zeta_{A, \Lambda}^{\prime \prime}\left(s_{0}\right) \neq 0$ then by Proposition $2.9 s_{0}$ is an interior point of a fundamental domain $\Delta$ of $\zeta_{A, \Lambda}^{\prime}(s)$. Since $\lim _{\sigma \rightarrow+\infty} \zeta^{\prime}(\sigma+i t)=0$ the function $\zeta_{A, \Lambda}^{\prime}(s)$ maps any unbounded curve $\Gamma$ originating at $s_{0}$ and such that $\lim _{\sigma \rightarrow+\infty} \zeta^{\prime}(\sigma+i t)=0$, for $\sigma+i t \in \Gamma$ onto a closed curve $\gamma$ passing through the origin. We can take $\Gamma$ completely included in the fundamental domain $\Delta$. Suppose that $z_{1}$ is a point interior to $\gamma$ and let $s_{1} \in \Delta$ be such that $\zeta^{\prime}\left(s_{1}\right)=z_{1}$. If $s_{2}$ is an arbitrary point of $\Delta \backslash \Gamma$ we can connect $s_{1}$ and $s_{2}$ by a curve $\Gamma_{1} \subset \Delta$ not intersecting $\Gamma$. Then the image of $\Gamma_{1}$ by $\zeta^{\prime}(s)$ cannot intersect $\gamma$. But if $z_{2}=\zeta^{\prime}\left(s_{2}\right)$ was outside $\gamma$, this is a contradiction, hence we cannot have $\zeta_{A, \Lambda}^{\prime \prime}\left(s_{0}\right) \neq 0$.

On the other hand, if $\zeta_{A, \Lambda}^{\prime \prime}\left(s_{0}\right)=0$ then we still get an indetermination of the form $0 / 0$ and the l'Hospital rule can be applied again giving

$\varphi\left(s_{0}\right)=\lim _{s \rightarrow s_{0}} \frac{\zeta_{A, \Lambda}^{\prime \prime \prime}(s)}{\zeta_{A, \Lambda}^{\prime \prime}(s)}$. By using the same argument as in the previous case we infer that $\zeta_{A, \Lambda}^{\prime \prime \prime}\left(s_{0}\right)$ cannot be different of zero and the process continues indefinitely. Hence all the coefficients of the Taylor expansion of $\zeta_{A, \Lambda}(s)$ at $s_{0}$ cancel and then $\zeta_{A, \Lambda}(s)$ is identically equal to zero in a neighborhood of $s_{0}$, which is impossible.

The final conclusion is that $\zeta_{A, \Lambda}(s)$ cannot have any multiple zero and the only zeros of $\varphi(s)$ are the zeros of $\zeta_{A, \Lambda}^{\prime}(s)$, which as we have seen cannot be at the same time zeros of $\zeta_{A, \Lambda}(s)$.

Remark: We have seen that the Dirichlet functions $f_{\tau}(s)$ defined in [3] have double zeros for some values of $\tau$. They are not Euler product functions. One might think that despite of this fact, taking the ratio $f_{\tau}^{\prime}(s) / f_{\tau}(s)$ we could deal with a function $\varphi_{\tau}(s)$ as the function $\varphi(s)$ above. However, when trying to define $\varphi_{\tau}(s)$ at $s_{0}$ we realize that this is impossible since $f_{\tau}(s)$ and all its derivatives cancel at $s_{0}$, which is absurd. Indeed, $f_{\tau}(s)$ should then be identically zero in a neighborhood of $s_{0}$ and by the uniqueness theorem of analytic functions throughout its domain and this is obviously not the case. Therefore the condition on $\zeta_{A, \Lambda}(s)$ to be an Euler product function is essential.

We emphasize also the fact that the Dirichlet L-functions, and in particular the Riemann Zeta function, are Euler product Dirichlet functions and therefore they cannot have multiple zeros. For a reader willing to aquire more information on this topic we recommend Chapter 11, Dirichlet series and Euler products of the monograph Multiplicative Number Theory by H. Montgomery and R. C. Vaughan.

\section{Conclusion}

Theorem 4.1 gives the solution of the outstanding problem, whether the 
Dirichlet functions admit or not multiple zeros. The answer is negative and it concerns a wider class of functions, namely those obtained by analytic continuation across the converging line of general Dirichlet series which can be written as Euler products.

\section{Acknowledgements}

We thank Aneta Costin for her support with technical matters.

\section{Conflicts of Interest}

The author declares no conflicts of interest regarding the publication of this paper.

\section{References}

[1] Ghisa, D. (2019) Fundamental Domains of Dirichlet Functions. In: Mladenov, I.M., Pulov, V. and Yoshioka, A., Eds., Geometry, Integrability and Quantization, Avangard Prima, Sofia, 131-160. https://doi.org/10.7546/giq-20-2019-131-160

[2] Ghisa, D. (2017) The Geometry of the Mappings by General Dirichlet Series. APM, 7, 1-20.

[3] Ahlfors, L.V. (1979) Complex Analysis. McGraw-Hill, New York. 